\title{
MDCT Scan Evaluation of Abdominal Lymphoma
}

\author{
Tushar Kalekar ${ }^{1}$, Pooja Shriramwar ${ }^{2}$ \\ ${ }^{1}$ Professor, Department of Radiodiagnosis, Dr D Y Patil Medical College, ${ }^{2}$ Radiologist, Megavision Scan Centre, Pune, \\ Maharashtra, India
}

Corresponding author: Dr Tushar Kalekar, 11, Suvarna Society, Gagangarima near Khadki Railway Station Pune, Maharashtra, India

DOI: 10.21276/ijcmsr.2018.3.2.31

How to cite this article: Tushar Kalekar, Pooja Shriramwar. MDCT scan evaluation of abdominal lymphoma. International Journal of Contemporary Medicine Surgery and Radiology. 2018;3(2):B127-B132.

\section{A B S T R A C T}

Introduction: Accurracy of CT in the staging of patients with lymphoma is widely recognized, and it is now the first investigation of choice in the initial staging and follow up of these patients. The current research aimed to study the role of CT scan of the abdomen in staging of the different types of the lymphoma, to study the differences of the involvement pattern and morphology among the different types of the lymphoma and to assess the role of the CT scan to characterize the morphology of primary lymphomas involving the hollow and solid abdominal viscera.

Material and methods: The study was a retrospective survey from 2014 to 2015. CT scan abdomen was performed in 80 patients of histological proved lymphoma; All the scans were performed using 128 slice CT scanner

Results: In this retrospective male preponderance study of 80 patients with proven lymphoma, positive findings were seen in $57(71.2 \%)$ patients, with $46 \%$ of HD and $84 \%$ of NHL patients showed intraabdominal involvement CT accurately detected nodal and extranodal sites of involvement,

Conclusion: Lymphnode involvement was the most common manifestation of lymphoma and it was seen in $66 \%$ of all the patients. CT showed the exact sites, the pattern of involvement, the morphological features and associated complications like encasement of the vessels, hydronephrosis, biliary obstruction, bony erosion, extramedullry cord compression etc. in both HD and NHL. Extranodal involvement was detected in -- patients. The involvement of liver was seen in $28 \%$, spleen in $26.3 \%$, GIT in $14 \%$ and renal involvement was seen in $7 \%$ of the patients with positive findings. Other sites of involvement include pancreas, psoas muscle and vertebrae.

Key Words: MDCT Scan, Abdominal Lymphoma

\section{INTRODUCTION}

Lymphomas are malignant tumors of lymphoreticular origin i.e. from lymphocytes and histiocytes and their precursor cells. Clinically and pathologically, lymphomas are quit heterogenous. However, two distinct clinic pathologic groups are routinely distinguished; Hodgkin's disease (HD) ${ }^{1,15}$ and Non Hodgkin's lymphoma (NHL). ${ }^{4}$

They are one of the common neoplasms of the patients in the young age group. Accurate staging of the patients is very important in planning appropriate therapy as advent of newer chemotherapeutic regimens and radiotherapy planning has improved the outcome with aim of achieving total cure rather than palliative cure. A series of examinations were performed for this purpose

In the past lymphography was performed as primary investigation toasses theretroperitoneallymphadenopathy, ${ }^{11,14}$ It is highly accurate in the detection of macroscopic nodal abnormalities. The limitations are few, although the nodes above cisterna chyli in the root of mesentery and in the hepatic or splenic hila are not demonstrated Exploratory laporatomy ${ }^{6}$ was undertaken to further stage patients when bone marrow biopsy did not reveal any tumor. Ultrasound is a useful survey technique for bulky retroperitoneal lymphadenopathy and can also detect isolated enlarged nodes or groups of nodes in the other areas. But main disadvantages are the operator dependency and sub optimal evaluation due to excessive bowel gases and obesity. Computed tomography (CT) is a more accurate method in this regard and in addition lymphomatous involvement of other abdominal viscera including liver, spleen, gastrointestinal tract, genitourinary system can be detected earlier.

It presents a cross-sectional picture of the abdomen which can be used in planning of radiation therapy ports. ${ }^{4}$ So CT forms an essential component of staging procedure. Administration of oral contrast prior to $\mathrm{CT}$ scan allows good visualization of the bowel loops and separates them from retro peritoneal and mesenteric nodes as well as vessels. Intravenous administration of water soluble non ionic contrast permits visualization of vascular structures which is essential to separate vessels from lymphnodes.Different patterns of enhancement helps in detection of lymphomatous deposits in the various abdominal organs.

CT is also the most acceptable and used modality for follow up of patients with Lymphoma. ${ }^{2.8}$

The current research aimed to study the role of CT scan of the abdomen in staging of the different types of the lymphoma, to study the differences of the involvement pattern and 
morphology among the different types of the lymphoma and to assess the role of the CT scan to characterize the morphology of primary lymphomas involving the hollow and solid abdominal viscera and the roles of CT scan in follow up of the patients.

\section{MATERIAL AND METHODS}

The study was a retrospective survey from 2014 to 2015. CT scan abdomen was performed in 80 patients of histological proved lymphoma. All the scans were performed in our institution after obtaining permission from hospital ethics committee.

\section{Study population}

A total of 80 subjects were included irrespective of their age and sex with following inclusion and exclusion criteria.

Inclusion criteria: Patients with histopathological diagnosed cases of lymphoma were included in the study

Exclusion criteria: Patients with contrast allergy and pregnancy.

\section{Equipment used}

CT Machine: 128 slice PHILIPS BRILLIANCE.

\section{Method}

Complete history was taken and histopathological report was noted. Other laboratory and haematological tests were observed.

\section{Technical parameters are as follows}

Field of view - $300 \mathrm{~mm}$, Length- $240 \mathrm{~mm}$.

Detector configuration (in mm) $-16 \times 1.5 \mathrm{~mm}$

Pitch factor - 0.938

Gantry rotation time $-0.5 \mathrm{sec}$

Reconstructed slice thickness $-1.0 \mathrm{~mm}$

Increment- $1.0 \mathrm{~mm}$

Tube current (in eff. $\mathrm{mAs}$ ) - 200, kV- 120

Contrast material: Volume $-2 \mathrm{ml} / \mathrm{kg}$

- Concentration- $300 \mathrm{mg} / \mathrm{ml}$

- Injection rate $-2 \mathrm{ml} / \mathrm{s}$

\section{Technique}

Prior to the procedure, all patients are instructed to be fasting for $4 \mathrm{hrs} .800 \mathrm{ml}$ of oral contrast is given to the patient over 45 minutes before the scan, with another $200 \mathrm{ml}$ just before taking the patient on for scanning. Contiguous $1 \mathrm{~mm}$ thick plain scans with an interval of $1 \mathrm{~mm}$ are performed for the liver, followed by post contrast scan of $1 \mathrm{~mm}$ thickness at an interval of $1 \mathrm{~mm}$, from the domes of the diaphragm to the pubic symphysis.

\section{RESULTS}

In the retrospective study of 80 patients with histological proven diagnosis of lymphoma were reviewed, and the following data was obtained.

1. Total number of patients

2. Total number of male patients

3. Total number of female patients 27

Male: Female ratio

\section{Findings at initial CT scan}

Out of 80 studied cases 23 were normal and 57 showed

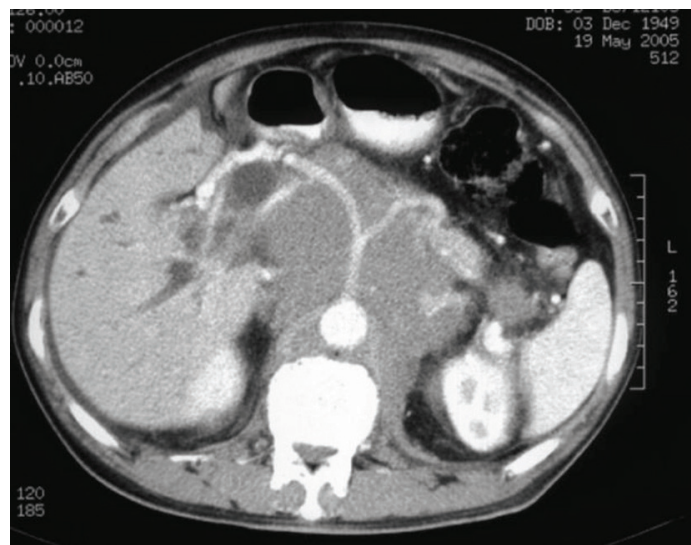

Figure-1: Large homogenous retroperitoneal lymphnode mass encasing vessels

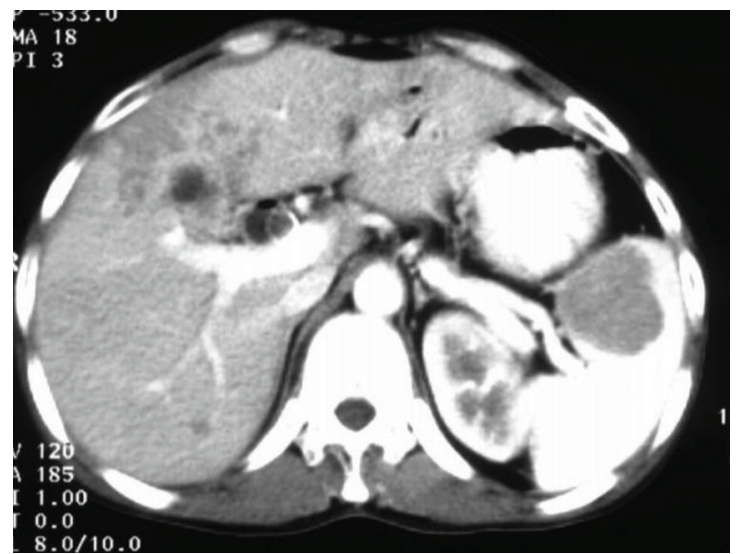

Figure-2: Involvement of liver and spleen in Hodgkin's disease

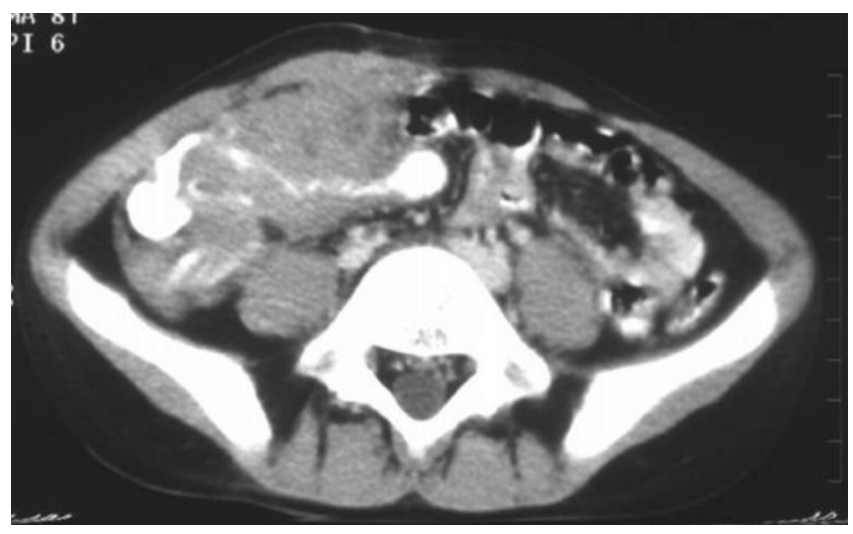

Figure-3: Involvement of caecum with circumferential wall thickening in NHL

abnormal finding in the abdomen. Amongst positive cases the 13 were of Hodgkin's disease and 44 were of Non Hodgkin's lymphoma. Positive findings are divided into two categories one with lymphnode involvement and other category of extra nodal abdominal organs involvement.

\section{Hodgkin's lymphoma}

Lymphnode involvement in Hodgkin's disease was observed in only 8 patients. It is classified in less than $2 \mathrm{~cm}$, more than $4 \mathrm{~cm}$ and between 2 to $4 \mathrm{~cm}$ as mild, gross and intermediate enlargement. Location wise enlarged lymphnodes seen in retroperitoneum in six patients, mesentery in one and iliac 


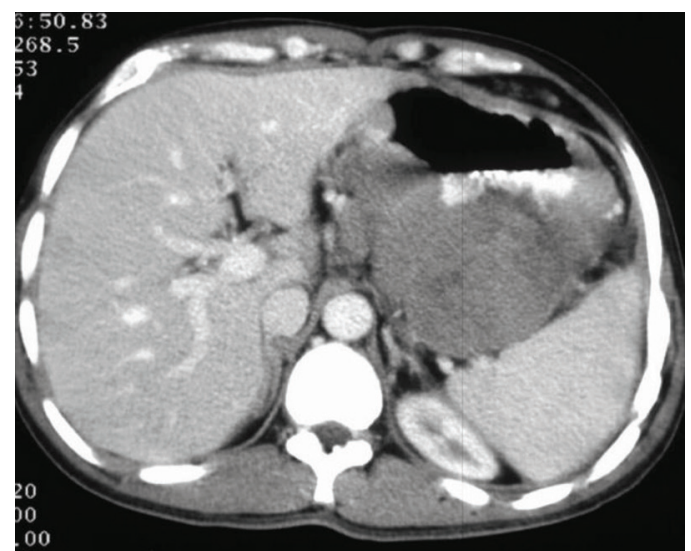

Figure-4: Primary stomach lymphoma with homogenous mass like involvement in NHL

region in one patient. Less than $2 \mathrm{~cm}$ size of lymphnode is observed in three cases, more than $4 \mathrm{~cm}$ was observed in two patients and three patients showed lymphnode enlargement between 2-4 cm. Most of the lymphnodes are homogenous, solid enhancement and only one showed areas of necrosis and heterogenous enhancement.

Extra nodal involvement observed in five patients. The involvement of the liver, spleen and kidney was observed in these patients in the form of organ enlargement and focal disease in the form from hypodense enhancing /non enhancing lesions. Two patients showed involvement of liver, two showed spleen involvement and one shows kidney involvement. Out of these one patient showed involvement of liver, spleen and kidney and not as isolated involvement

\section{Non Hodgkin's lymphoma}

Out of total 44 patients with NHL, 30 patients showed enlargement of lymphnodes. 24 patients showed retroperitoneal lymphnode enlargement, 9 showed mesenteric lymphnode enlargement, 16 patients had iliac lymphadenopathy, 2 showed enlarged obturator and 4 showed retrocrural enlarged lymph nodes

10 patients showed lymphnode enlargement less than $2 \mathrm{~cm}$, 13 patients showed lymphnode size between $2-4 \mathrm{~cm}$ and 7 patients showed lymphnode size more than $4 \mathrm{~cm}$. Most of the lymphnodes are homogenous, solid enhancement and few showed areas of necrosis and heterogenous enhancement. Most of the lymphnodes are conglomerated and only 4 cases showed discrete lymphnode enlargement

Extranodal involvement observed in 14 patients. The involvement of the liver, spleen, gastrointestinal tract, kidneys and other organ involvement was observed in these patients in the form of organ enlargement and focal disease in the form from hypodense enhancing / non enhancing lesions. These extranodal site involvements was observed in associated with other organ involvement rather than isolated finding in almost all the cases. Ten patients showed involvement of liver, ten showed spleen involvement, 8 showed GIT involvement and six showed kidney involvement. Six patients showed involvement of other organs. In two cases there is involvement of the pancreas, in one peritoneal involvement was observed and three cases showed involvement of the bones and muscles

\section{DISCUSSION}

In this study of 80 patients, the age group ranged from 3 years to 78 years. The peak incidence of HD was in second decade, with 10 patients (35.7). There is second small peak observed in the fourth decade with 4 patients $(14.2 \%)$ So there is bimodal peak observed in the second and fourth decade. The peak incidence of NHL, in the series was in fifth decade, with 14 patients in this age group (29.6\%) This corroborates with the age related incidence of lymphoma in the literature. There was male predominance in the study (male to female ratio was $1.96: 1)$.

23 cases of HD and 57 cases of NHL were studied in the study. The commonest histological subtype of HD was Nodular sclerosis accounting for $46.4 \%$ of the cases.

In $57(71.2 \%)$ of the 80 patients, the initial CT scan of abdomen showed evidence of involvement by lymphoma at one or more sites. In 23 patients CT scan showed normal findings (28.7\%). The CT scan was normal in 15 out of 28 patients of HD (53.5\%) and it was normal in only 8 patients of NHL (15.3\%) The commonest abnormality found was enlarged lymph nodes. (66.6\%)fig 1. These findings are consistent with the previous literature studies showing that the abdominal involvement is more common in Non Hodgkin's lymphoma than HD.

\section{Lymph nodes}

Abdominal lymph node enlargement ( $>10 \mathrm{~mm}$ in diameter, $>6 \mathrm{~mm}$ retrocrural and $>15 \mathrm{~mm}$ pelvic) was initially present in $38(66.6 \%)$ of the 57 patients with evidence of positive findings. Enlarged nodes were seen in the upper abdomen only from diaphragm to aortic bifurcation) in 14 patients, in the abdomen and pelvis in 24 patients, and in the pelvis in 6 patients. Enlarged nodes in the abdomen and pelvis are seen in 22 out of 30 patients of NHL and in only 2 out 8 cases of HD. This is consistent with the literature studies that NHL $^{11,15}$ shows wide spread involvement as compared to $\mathrm{HD}$ which presents as localized disease. 8 out of 28 patients of $\mathrm{HD}(28.5 \%)$ as compared to 30 patients out of 52(57.7\%) of NHL.These finding are consistent with Castellino $\mathrm{R} \mathrm{A}^{6}$ who showed $24 \%$ of HD and $48 \%$ of NHL showed retro peritoneal lymph node involvement. The mesenteric involvement was seen in only 1 out of 28(3.5\%) cases of HD as compared to 9 out of $52(17.3 \%)$ cases of NHL. These findings were similar to Blackledge and Castellino et $\mathrm{al}^{6}$ who showed that mesenteric lymph node involvement was more common, in NHL than HD.Also the involvement of the different nodal sites like portal, peripancreatic, celiac, which seen predominantly in NHL. The retrocrural,splenic,renal hilar enlargement was seen only in NHL cases (fig 1).

In 20 of the 38 patients with nodal enlargement, CT scan showed evidence of lymphomatous involvement of one or more of the following sites, GI tract (4 patients), liver (10), spleen (10), and omentum (1).In one patient, the only extra nodal site of involvement was the epidural space. 5 out of 8 patients of HD and 15 out of 30 patients of NHL with lymph node involvement show extra nodal involvement.

The involvement of the retro peritoneal lymph nodes in Para aortic region was most common; it was seen in 30 patients out of 38 who showed lymph node involvement (78.9\%) 
It was seen equally common in both HD (75\%) and NHL (80\%). In 13 patients with enlarged abdominal lymph nodes, the diameter of the largest node was upto $2 \mathrm{~cm},(34.2 \% \%)$, $2-4 \mathrm{~cm}$ in 18 patients $(47.3 \%)$, and $4-10 \mathrm{~cm}$ or more in 7 patients (18.4\%) (fig 1$)$.

In 24 (63.1\%) of the 38 patients, all nodes were homogenous, usually more dense than the muscle. In 13 patients (34.2\%) the nodes had central low density, The nodes with central necrosis ranged in diameter from 4 to $10 \mathrm{In}$ one patient there was evidence of calcification. Evidence of necrosis is seen in 11 out of 30 cases (36.6\%) of NHL showing lymph node involvement as compared to only 2 out of 8 in patients of $\mathrm{HD}(25 \%)$. This showed that the evidence of necrosis is more common in bulky nodes and so in NHL cases than HD who showed smaller and homogenous nodes.

Calcification in patients with lymphoma occurring before therapy is rare as opposed to that in lymphoma after therapy. It occurred in our patients more often in the mediastinum, in patients with non-Hodgkin's lymphoma rather than in patients with Hodgkin's lymphoma, and only in patients with the aggressive type of disease.

\section{Liver}

In this study, the involvement of liver by lymphoma was seen in 16 cases (20.0\%) out of 80 in the form of diffuse enlargement and or focal lesions, these results were comparable to the study of Zoronza et $\mathrm{al}^{16}$ who have found hepatic involvement in $11.5 \%$ of cases. Diffuse enlargement with or without focal lesions is seen in 16 patients with positive findings (28.0\%). Diffuse enlargement without focal lesions is seen in 9 patients (15.7\%) Focal lesions, varying in size from solitary to multiple were seen in 9 out of 57 patients with positive findings (15.7\%). In all cases the focal lesions were less dense than the liver parenchyma (fig 2). Only 5 patients show focal lesions without evidence of enlargement (8.7\%). In the absence of focal lesions the enlargement of the liver was mild (liver span less than $18 \mathrm{~cm}$ ). In 4 of 80 patients with focal lesions showed marked hepatomegaly (more than $18 \mathrm{~cm}$ ) 6 out of 28 cases of HD showed hepatic involvement $(21.4 \%)$ as compared to 10 out of 52 patients with $\mathrm{NHL}(19.2 \%)$ Diffuse enlargement without focal lesions is seen in $4(50 \%)$ patients of HD with hepatic involvement as compared to 3(33.3\%) of NHL cases

Out of 16 cases with hepatic involvement by lymphoma also showed splenic involvement in 12 cases, (75\%) this correlates with the study of Strijk et $\mathrm{al}^{20}$, where the involvement of the was always accompanied by splenic disease. In 11 out of 16 (68.7) patients with liver involvement showed evidence of lymphadenopathy. In one patient there is associated IHBR dilatation due to portal adenopathy.

\section{Spleen}

Splenic involvement was seen in $15(18.75 \%)$ out of 80 and $26.3 \%$ of all the patients with positive abdominal findings. with 9 patients showing enlargement only without evidence of any focal lesions, 2 patients showed focal lesions with splenomegaly (fig. 2). Focal splenic lesions were seen in patients that varied from solitary to multiple in numbers These results were comparable to the study of Castellino et al ${ }^{7}$, that showed splenic abnormalities with CT in $14 \%$ of cases and Pond et al 35 detected splenic abnormalities in $15.1 \%$ of the cases. Previous studies have shown that the detection of splenic disease by $\mathrm{CT}$ is difficult due to the miliary pattern of involvement.

Splenic involvement was seen in $5(17.8 \%)$ out of 28 cases of $\mathrm{HD}$ and 4out of 5 patients showed diffuse enlargement without any focal lesion. 10 patients of NHL showed splenic involvement (19.2\%) and only 3 patients showed diffuse enlargement and focal lesions are seen in 5 patients.(fig 2)) The degree of splenic enlargement was mild $(12-15 \mathrm{~cm})$ in 5 patients and moderate in 4 patients $(15-20 \mathrm{~cm})$.In all the 4 patients with moderate splenomegaly there is associated hepatomegaly

In 12 patients $(80 \%)$ with splenic involvement there is associated lymphnode involvement.

\section{Kidney}

The CT scan showed evidence of renal involvement by lymphoma in $4(5 \%)$ of all the patients and. In 3 case there was evidence of diffuse renal lymphoma seen. In one patient there are focal lesions. The results are comparable to Cohan et $\mathrm{a}^{14}$ who found $7 \%$ incidence of renal involvement in malignant lymphoma. The renal nodules were less dense than the adjacent renal parenchyma on post contrast scans. 3 out of 4 patients (75\%) were of NHL and one patient of HD showed diffuse enlargement of both kidneys. In all the patients with renal lesions CT scan showed other sites of extra nodal involvement. Perirenal lymphomatous involvement was seen in one patient of NHL.

Gastrointestinal tract

GIT involvement was seen in $8(10 \%)$ of the CT scans. Sites of involvement included the stomach 3 (fig 4) (37.5\%) patients, followed by small intestine(ileo-cecal region) fig.4 in 3 cases (37.5\%)and the large bowel in 2 patients (25\%). The results were similar to the study of Lewin K J et al ${ }^{26}$ who showed the stomach as the commonest site of GIT followed by small and large intestine. and appendix. All the patients were of diffuse Non Hodgkin's lymphoma and the age group ranged from 35 to 75 years.

The CT findings showed circumferential wall thickening as commonest pattern in 6 cases. Focal eccentric wall thickening and a mass in 2 patients (ileo-cecal region)(fig 4) This morphological pattern was similar to that described by Dodd et $\mathrm{al}^{27}$, who found circumferential wall thickening more than $1.5 \mathrm{~cm}$ in most of the patients (fig 4$)^{7}$

In 5 of the 8 cases $\mathrm{CT}$ also showed evidence of lymphnode involvement (62.5\%) associated in 2 cases focal lesions in the solid viscera. The results are comparable to the study of Buy et $\mathrm{al}^{2}$ who found associated lymphadenopathy in $92.5 \%$ of the patients.

Pancreas

Pancreatic involvement was seen in 2 patients (2.5\%) all of NHL type (3.5\% of NHL) All the cases showed extensive retro peritoneal and mesenteric lymphadenopathy.

The pattern of pancreatic involvement in the form of infiltration from adjacent lymphnodes in all the cases correlates with the study of Glazer et $\mathrm{al}^{5}$

\section{Peritoneum}

Ascites was seen in only one patient of the abdominal 
lymphoma cases. Omental and peritoneal infiltration was seen in the 2 patients. All these patients were of NHL, high grade type and there was associated extensive retroperitoneal lymph node enlargement.

Musculoskeletal involvement

CT showed lymphomatous involvement of the muscle in 2 patients (2.5\%). Marked enlargement of the psoas muscle with heterogenous density was seen In one patient eccentric soft tissue infiltration of the epidural space of the lumbar spine was observed All the patients were of NHL high grade $(3.4 \%)$ and associated with extensive retroperitoneal lymphadenopathy and focal lesions were present in liver. (table 3) The findings in our study correlate with that of Glazer et al 22, where the incidence of muscle involvement was $4 \%$,all the patients having associated retro peritoneal lymphadenopathy

Follow up CT scan were available in in 20 patients with 15 patients showing regression of the disease (75\%), 4 patients showed progression of the disease (20\%)and one patient showed no change in the disease status since the previous scan.(5\%). The CT findings corroborated with clinical features in all the patients (100\%) These findings correlate with the study of Oliver et $\mathrm{al}^{31}$ with $\mathrm{CT}$ scan showing an excellent correlation with clinical status of the patient.

Out of 4 patients with disease progression two patient showed enlargement of the lymph node size and involvement of the other sites that were initially not involved and 2 patients showed deposits in the liver and spleen with enlargement of their size which was not seen initially.

All the patients with regression of the disease showed reduction of the lymphnode size or disappearance of the lymphnode mass and decrease in the attenuation of the lymphnode and evidence of the calcification. Calcification after treatment was seen in 4.

The patient with no change in the disease status was a case of HD and showed small lymphnodes in the upper retroperitoneum as were seen in the initial scan.

\section{CONCLUSION}

The accuracy of CT in the staging of patients with lymphoma is widely recognized, and it is now the first investigation of choice in the initial staging and follow up of these patients Staging laparotomy involving splenectomy and biopsy of all lymphnode sites was formerly gold standard for staging of intraabdominal lymphoma, but is now rarely performed. Most centers now rely on biopsy of one group of nodes for diagnosis and stage the patient based on imaging with CT and hematological assessment including bone marrow biopsy. In this retrospective male preponderance study of 80 patients with proven lymphoma, positive findings were seen in $57(71.2 \%)$ patients, with $46 \%$ of $\mathrm{HD}$ and $84 \%$ of NHL patients showed intraabdominal involvement $\mathrm{CT}$ accurately detected nodal and extra nodal sites of involvement. So CT scan is the essential investigation modality in all patients with lymphoma as it can detect the intra abdominal disease

\section{REFERENCES}

1. Earl H.M.et al. Computerized Tomographic abdominal scanning in Hodgkin's Disease, Clinical Radiology
1980;31(1):149-153.

2. Buy J N., Moss A A. Computed Tomography of gastric lymphoma, AJR 1982;138(2):859-865.

3. Glazer et al. CT of renal and perirenal non-Hodgkin lymphoma. AJR Am J Roentgenol. 1982;138(6):1101-5

4. Pond G D. Potential pitfall in CT and sonographic evaluation of suspected lymphoma.AJR Am J Roentgenol. 1982;139(3):606-7.

5. Glazer et al. Computed tomography of renal lymphoma with ultrasound correlation. J Comput Assist Tomogr. 1983;7(2):245-50.

6. Castellino R A, Hoppe R. T. et al. Computed Tomography, Lymphography and Staging Laparotomy: Correlation in initial staging of Hodgkin's Disease. AJR 1984;143(5):37-41.

7. Strijk et al. Distribution of abdominal and pelvic Hodgkin disease: implications for CT scanning J Comput Assist Tomogr. 1985;9(3):463-5.

8. Bragg D G., Colby T V., et al. New concepts in Non Hodgkin's Lymphoma: Radiologic implications, Radiology 1986;159(4):289-304.

9. Pera A,Capek $\mathrm{M}$ et al. Lymphography and CT in the follow up of patients with lymphoma, Radiology 1987;164(5):631-633.

10. Pond G D., Castellino R. A. et al. Non Hodgkin's Lymphoma; Influence of lymphography, CT, and Bone marrow biopsy on staging and management, Radiology 1989;170(3):159-164.

11. Jing B. Diagnostic Imaging of abdominal and pelvic lymphadenopathy in Lymphoma, RCNA, 1990;28(4):801-832.

12. Einstein D M., Singer A.A, et al. Abdominal Lymphadenopathy: Spectrum of CT findings, Radio graphics 1991;11(5):457-472.

13. Cheryman G. The Lymphatic system. Textbook of Radiology and Imaging, David Sutton, $5^{\text {th }}$ Edition: 731752.1992 .

14. Cohan et al. Non Hodgkin's Lymphoma: Abdominal CT findings in 112 patients, AJR 1993;160:1133-1139.

15. Paul C, Scott P., et al. Abdominal staging of thoracic Hodgkin's Disease: CT-Lymphangiography-GA67 Scanning correlation, Radiology 1993;187(6):381-386.

16. Hancock SL et al. Computed tomography assessment of splenic size as a predictor of splenic weight and disease involvement in laparotomy staged Hodgkin's disease. Int J Radiat Oncol Biol Phys. 1994;28(1):93-9.

17. Jacobs JE, Salhany KE et al. Omental caking in Hodgkin's disease Computed tomography findings. Clin Imaging. 1996;20(4):253-5.

18. .Cheremisin VM, Mazurov VI. Computed tomography diagnosis of extranodal manifestations in malignant non-Hodgkin's lymphoma] Vestn Rentgenol Radiol. 1996;(1):30-4.

19. Naik K S., Spencer J A et al. Staging Lymphoma with CT: Comparison of Contiguous and Alternate $10 \mathrm{~mm}$ Slice Technique, Clinical Radiology, 53:523-527,1997.

20. Strijk et al. CT of small-bowel lymphoma in immunocompetent patients and patients with AIDS: comparison of findings. AJR Am J Roentgenol. 1997;168(3):675-80.

21. Johnson K A., Tung K., et al. Imaging of Burkitt's 
and Burkitt like Lymphoma, Clinical Radiology 1998;53(4):835-841.

22. Lewin $\mathrm{K} \mathrm{J}$ et al et al. Primary non-Hodgkin's splenic lymphoma. Clin Radiol. 1998;53(2):137-42.

23. Oh YK, Ha CS et al. Stages I-III follicular lymphoma: role of CT of the abdomen and pelvis in follow-up studies. Radiology. 1999;210(2):483-6.

24. Glazer et al. Primary bilateral adrenal lymphoma Schweiz Med Wochenschr. 2000;130(31-32):1120-4.

25. Apter S, Avigdor A. et al. Calcification in lymphoma occurring before therapy: CT features and clinical correlation. AJR Am J Roentgenol. 2002;178(4):935-8.

26. Oliver et al. lymphoma musculoskeletal involvement. J Comput Assist Tomogr. 2003 May-Jun;8(3):4635Renno S I et al: Primary malignant lymphoma of uterine corpus: case report and review of the literature. Ann Hematol. 2002 Jan;81(1)

27. Dodd et al. Non-Hodgkin lymphoma and Hodgkin disease: coregistered FDG PET and CT at staging and restaging--do we need contrast-enhanced Radiology. 2004;232(3):823-9.

Source of Support: Nil; Conflict of Interest: None

Submitted: 05-05-2018; Accepted: 04-06-2018; Published online: 15-06-2018 